\title{
Propose A New Optimization Technique For Mobile IPv4
}

\author{
Awos Khazal Ali \\ Department of Computer Science $\backslash$ College of Education \\ University of Mosul
}

Received
$05 / 03 / 2012$

\section{Accepted \\ 25 / $06 / 2012$}

\section{الخلاصة}

يوفر بروتوكول الانترنيت الأصدار الرابع الذي يدعم الأجهزة الخلويـة اتصـال بشبكة

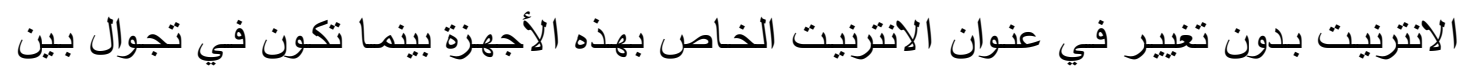

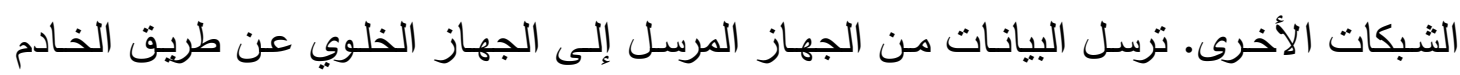

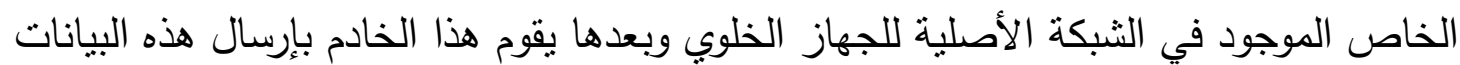

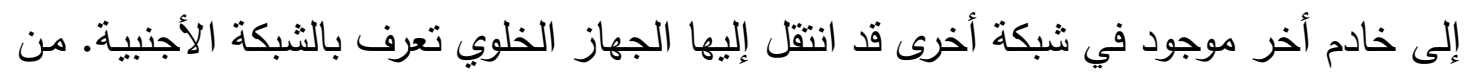

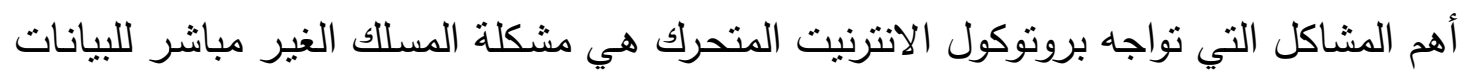

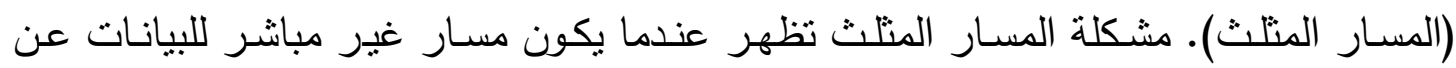

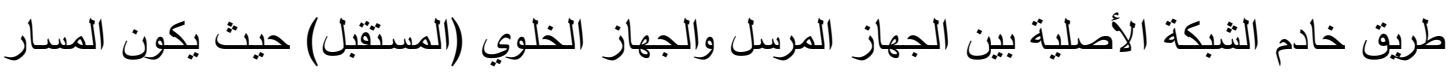

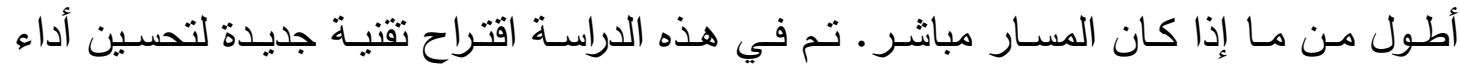

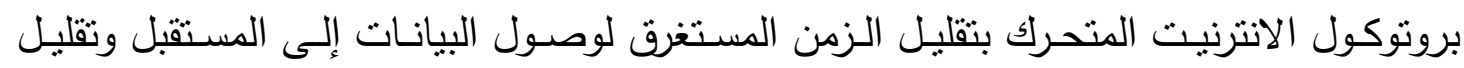

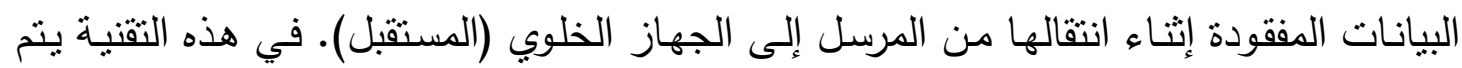

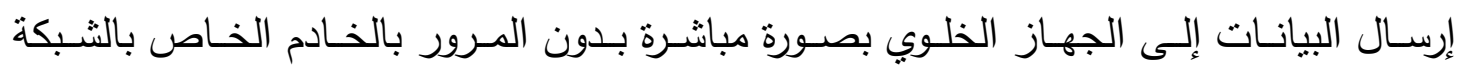

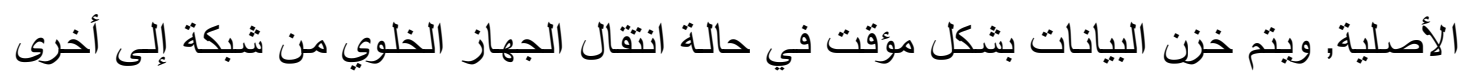
إلى إن تتم عملية الانتقال بنجاح ويحصل الجهاز الخلوي على العنوان الجديد ومن ثم ينت إعادة

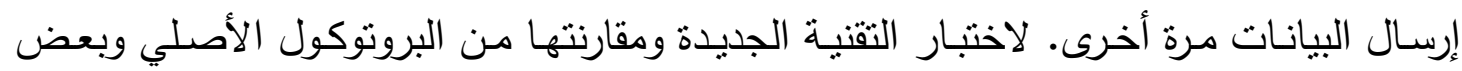

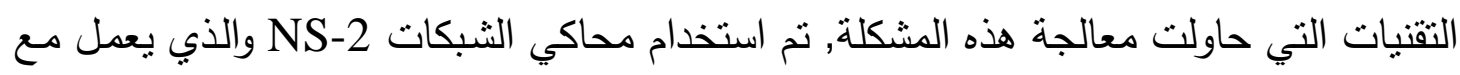

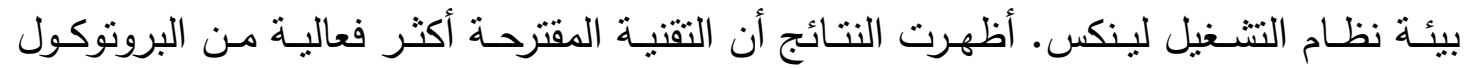
الأصلي والتقنيات الآخرى.

\section{ABSTRACT}

Mobile IPv4 is designed to allow the mobile nodes to have internet connectivity without changing their IP addresses while roaming between 
other networks. The packets sent from Correspondent Node $(\mathrm{CN})$ to a Mobile Node (MN) through the mobile node's Home Agent (HA), then the HA forward them to the MN's foreign network. One of the main problems that faced the original Mobile IP is the triangle routing problem. Triangle routing problem appears when the indirect path between $\mathrm{CN}$ and $\mathrm{MN}$ through the HA. The path is longer than the direct path. This paper proposes a new technique to improve the performance of the original Mobile IP by reducing the average delay and packet loss for all the packets transferred between the $\mathrm{CN}$ and the $\mathrm{MN}$. In this technique, the packets will delivered to $\mathrm{MN}$ directly without passing by $\mathrm{HA}$, and they will pauses in tunneling buffer till MN reach to new network and receive new address. To show the effectiveness of the proposed technique, it is compared with the original Mobile IP and another technique that try to solve the same problem. The simulation of the original Mobile IP and new technique is carried out using NS-2 simulator on Linux platform. The simulation results show that the proposed technique is more effective than the original Mobile IP and others.

\section{Introduction:}

In recent years the number of wireless and mobile devices that connected to the internet is growing in very fast way. The wireless mobile internet is an extension of the internet into the mobile environment, which gives users the ability to access to the internet services while they are on the move, on the other word, while they are roaming. Here the need of mobile IP (MIP) to proposed, to route the data packets from wired network to wireless network while mobile devices roaming between networks [1].

A mobile device will require a new point of attachment every time it moves to a new network, that's mean new IP address. For example in TCP/IP protocol if a host device changes its IP address, TCP will be unable to maintain the connection between the source node and destination node (Host) and for this reason, a work group within Internet Engineering Task Force (IETF) introduced an internet protocol called Mobile IP for supporting mobility in the internet [2].

Before describing the operation of mobile IP, some terminologies are needed to be explained first.

- Corresponding Node $(\mathbf{C N})$ : a node that sends data packets to mobile node $(\mathrm{MN})$, it may either be mobile or stationary [1].

- Mobile Node (MN): a movable node that changing its point of attachment to the internet and receives the data packets from $\mathrm{CN}$ [2].

- Home Network: mobile node's original point of attachment [2].

- Home Agent (HA): a router in home network that forward the data packets to MN whether MN away for home network or not [3].

- Foreign Network: the new network that mobile node (MN) visits [3].

- Foreign Agent (FA): a router in foreign network, provide MN with new address and later delivering data packets to MN [4]. 


\section{Mobile IP Operations:}

The concept of Mobile IP is simple. Mobile node (MN) can change its point of attachment in the specific network in internet to different network and still being identified by its original IP address, on the other words, MN still has connection with the source without terminate this connection when move from network to other. This process can be done by assigning two IP addresses to the MN. One IP address used when MN within home network, and second IP address is known as Care of address (COA) which used when MN within foreign network. So, allow a portable computer to be moved from one network to another without changing its IP address and without losing existing connections [2][3][4].

Mobile IP mechanism has three major components to establish connection between $\mathrm{MN}$ and HA or FA. These components are as follow:

A- Agent Discovery: the concept of agent discovery process is to let the mobile node $\mathrm{MN}$ know whether its in home network or in a foreign network by identifying whether the agent is a home agent or a foreign agent, this could be done by sending Agent Advertisements Message by home and foreign agents every second or once every few seconds. In addition, when $\mathrm{MN}$ receive agent advertisements message that sent by HA that's mean the MN in home network, or when MN receive this message that sent by foreign agent that's mean $\mathrm{MN}$ in foreign network [2][3].

B- Registration: when the $\mathrm{MN}$ in foreign network, $\mathrm{MN}$ initiates the registration process by sending registration request to foreign agent to know it's care of address (new address). Foreign agent just forwards this information about $\mathrm{MN}$ to home agent. On the other side, home agent approves the registration request and sends back a registration reply message to $\mathrm{MN}$ passing by foreign agent. During registration process, MN requests a routing service from foreign agent and renews a registration if it is expired. If a $\mathrm{MN}$ returns to its home network MN will deregister from foreign agent [2][4].

C- Tunneling: when registration process is complete; the data packets need to be delivered from home address to $\mathrm{MN}$ at its new care of address. This problem solved by tunneling the packets [4]. Tunneling packets means encapsulation the original IP packet in another IP header and send the packet to foreign agent, then delivered the original packet to $\mathrm{MN}$ can accomplished by removing the outer IP header. However, several tunneling techniques are proposed but the one that described above is widely used and is called IP-in-IP [5]. The following figure illustrates tunneling process. 


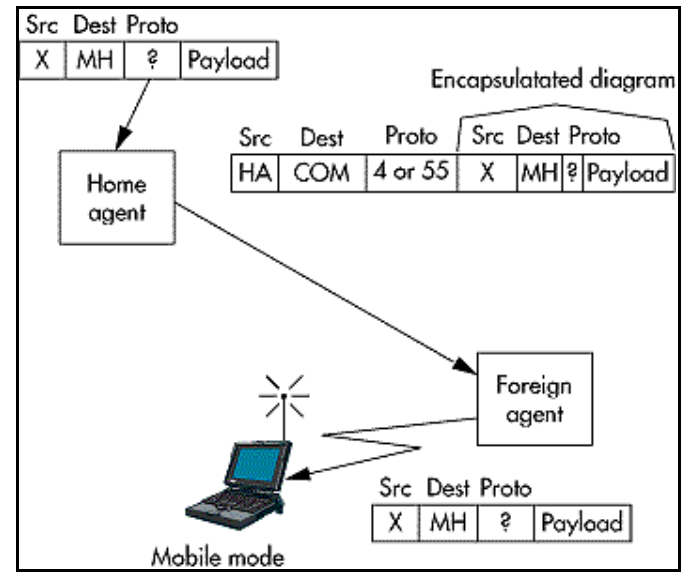

Figure 1: Tunneling Process [4]

\section{Triangle Routing Problem:}

In Mobile IP (MIP) any foreign network that a mobile node (MN) need to travel to must contain a router called a Foreign Agent (FA). The $\mathrm{MN}$ which is in a foreign network request a care of address (COA) from the FA, the MN contacts it's HA through the FA to notify the HA its new location (new COA). This care of address is the IP address of the Foreign Agent (FA). Moreover, when corresponding node $(\mathrm{CN})$ need to sent packets to the mobile node, they send first to Home Agent and encapsulate these packets after that they tunneled to the foreign agent [3][4].

The foreign agent will then decapsulate the packet and delivers it to the mobile node. To send a packet to the correspondent Node $(\mathrm{CN})$, the $\mathrm{MN}$ does not need to go through the HA but can directly send the packets to the $\mathrm{CN}$ using normal IP routing. The route of this traffic is look like as triangular in nature. The disadvantages of this scheme is that the routing is not optimal because the packets take the long path and traverse from sender $(\mathrm{CN})$ to mobile node instead of the direct path while it takes the direct path from the $\mathrm{MN}$ to the $\mathrm{CN}$. This creates a problem known as the triangular routing problem [5]. Figure 2 illustrates the triangle routing problem.

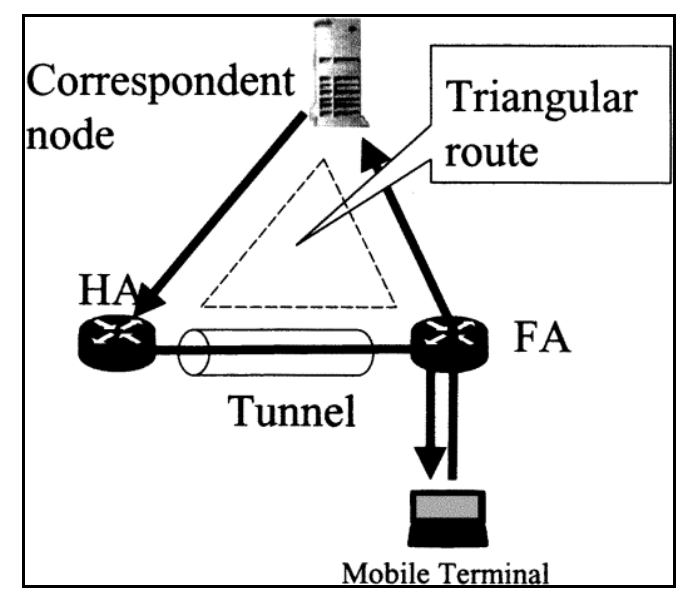

Figure 2: Triangle Routing Problem [5] 


\section{Related Work}

Since triangle routing problem cause a delay to the packet that need to reach the $\mathrm{MN}$ because it takes a long path from source to destination, and this issue is not acceptable for real time applications such as Voice over IP (VOIP). Several schemes have been proposed to solve triangle routing problem. These schemes will describe in brief way in the following subsections.

\subsection{Route Optimizations in Mobile IP (ROMIP)}

ROMIP protocol consist of four steps; Binding Cache Maintenance, Smooth Handoffs, Registration Key Management, and Special Tunnels. Before explaining these steps, two terms are needed to be explained:

Binding: contains the Mobile node's home address, care-of address and registration lifetime means how long mobility agents may use the binding [5].

Binding updates: The message that supplies a new binding to an entity that needs to know the new care-of address for a mobile node. It contains the mobile node's home address, new care-of address and a new registration life time [6].

ROMIP deals with correspondent nodes $(\mathrm{CN})$. Correspondent nodes must know the current location information of $\mathrm{MN}$, in other words, the $\mathrm{CN}$ will have to discover the MN's care-of-address, and maintain a Binding Cache for use in tunneling their own packets to the MN's careof-address directly. Whenever the care-of-address changes later, the correspondent nodes must update their Binding Cache. Route optimization accomplishes this by sending a Binding Update to the $\mathrm{CN}$. The production and consumption of these Binding Updates form the essential of the operation of the route optimization protocol [7].

- Binding Cache Maintenance: to deliver bindings to correspondent nodes, ROMIP defines four new messages sent to the same port as the base Mobile IP protocol. These messages are binding Warning (which tells correspondent nodes that it should get a new binding), binding Request (correspondent nodes asks for a new binding), binding Update (to the correspondent nodes) and binding Acknowledgement (correspondent nodes confirms that it has received the binding [6][7].

- Smooth Handoffs: when a binding Update is sent to the mobile node's previous foreign Agent and mobile node has moved to a new care-of address. In such case, previous foreign agent is responsible to deliver 
packets to its new care-of address. a Previous Foreign Agent Notification message has been proposed. A mobile node creates all the information needed by its new foreign agent to deliver a new authorized Binding Update to previous Foreign Agent. Previous Foreign Agent must send a confirmation back to new Foreign Agent. The Mobile node has to have an established security association with previous Foreign Agent before it creates an Update [7].

- Registration Key Management: to enable a smooth hand-off, mobile node needs to establish a security association with its foreign agents as described earlier. This is provided in ROMIP by using appropriate messages embedded in original Registration Request message. After the conclusion of Registration process when the mobile node receives the registration reply, these key establishment messages allow the distribution of registration key to both the mobile node and foreign agent [8].

- Special Tunnels: If the previous foreign agent has no fresh binding for the mobile node, special tunnels are used, which indicates to the Home Agent the need for special handling. Since mobile node's cache binding has expired, the previous foreign Agent will not be able to find the home address in the de-capsulated packet and therefore will not be able to send an IP packet back to the Home Agent. Instead of doing that, Foreign Agent encapsulates the IP packet to be sent to the Home Agent using its care-of address as Source IP address. Home Agent, after receiving this packet, compares the source address with the care-of address known in the binding created from the last registration. In case of a match, Home agent will not tunnel the IP packet back to the care-of address. If addresses do not match, then the decapsulated packet is re-tunneled and sent to the current care-of address known from the registration [8].

\subsection{Reverse Routing:}

Reverse routing was proposed as an alternative to ROMIP, tried to reduce the complexities of ROMIP. In order to make it more effective a new technique of updating the routing table was introduced. The mechanism of reverse routing is when A mobile node receives and sends IP packets usually on its home network. As it moves to a foreign network, it obtains a care-of address in foreign subnet. After receiving it, mobile node sends a registration message to the sender [8]. The registration message contains the mobile node's home address and care-of address and is routed from foreign network. When the registration message reaches the network where the sender is located, the router of the network intercepts the registration message, updates its routing table and the drops the registration message. Thus, all future messages for the mobile node 
from the sender will be routed according to the newly updated entry. Its called reverse routing as the registration message travels along in reverse direction from mobile node to the correspondent node. Figure 3 illustrates the Mechanism of reverse routing [9][10].

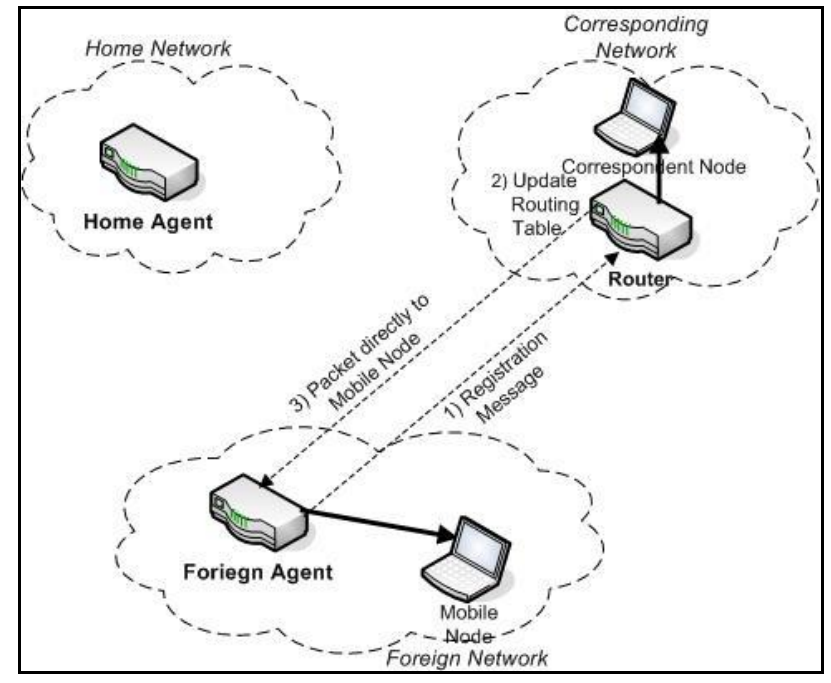

Figure 3: Mechanism of Reverse Routing [9]

\section{Proposed Work:}

This paper proposed a new enhancement technique to solve triangle routing problem in MIP to reduce packet delay to reach the MN (inspired from MIPv6) and packet loss during its traveling from $\mathrm{CN}$ to MN. The mechanism of proposed technique is when MN within it is HA area, it send and receive data packet in regular way (according to original MIPv4 operation). Whenever, MN travels from HA to FA, MN will request a new Care of Address (COA) by sending registration request to foreign agent. Foreign agent wills forwards this request to home agent, home agent will approves the registration request and sends back a registration reply message to $\mathrm{MN}$ passing by foreign agent. When $\mathrm{MN}$ receive its new $\mathrm{COA}$; $\mathrm{MN}$ will send its $\mathrm{COA}$ and it's HA address by second registration message to $\mathrm{CN}$ (sender). The last message will forward by foreign agent to $\mathrm{CN}$ directly. Moreover, when $\mathrm{CN}$ receive MN's registration message, $\mathrm{CN}$ will update its routing table (assuming $\mathrm{CN}$ will be mobile node or fixed node). Thus, all packets from $\mathrm{CN}$ to $\mathrm{MN}$ will be delivered directly according to new COA.

In order to reduce packet loss during MN's movements from home network to foreign network; this new technique propose adding Tunneling Buffer (TB) on home agent. So, when MN moves from $\mathrm{HN}$ to FN and MN still receive data packets from HA. TB will pause the packets that sending to $\mathrm{MN}$ until movement is complete. Thus, when $\mathrm{MN}$ reached a new FN; MN will request new address from HA. HA will send the packets that paused in TB to new MN's COA. Figure 4 illustrate the mechanism of proposed technique. 


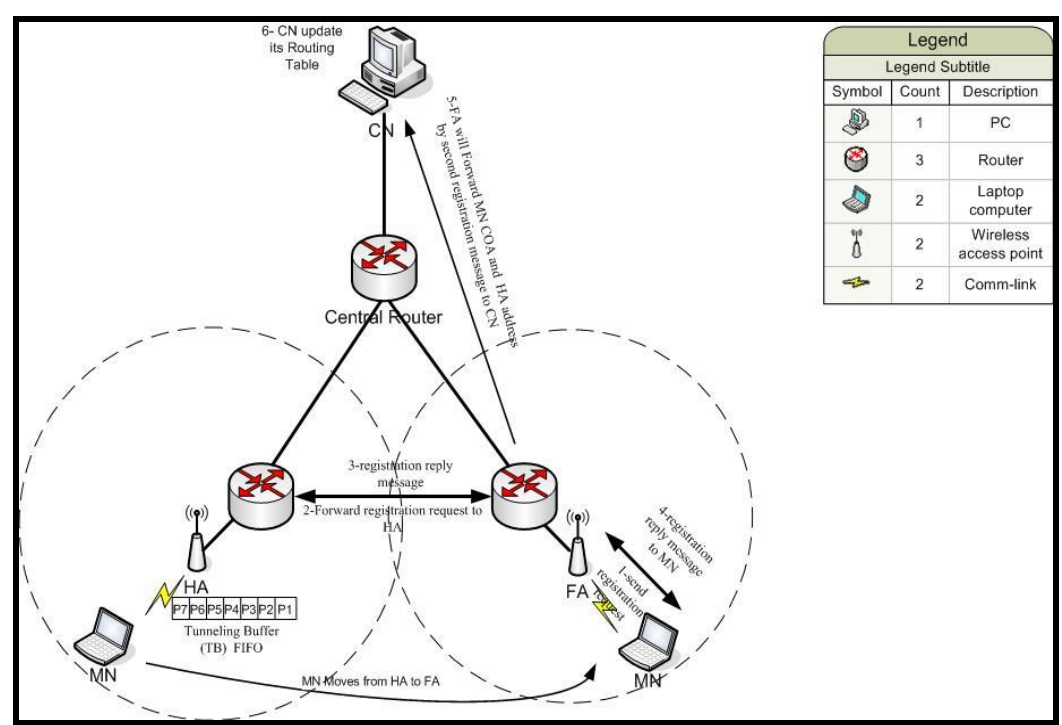

Figure 4: the mechanism of proposed technique.

\section{Simulation Scenario}

In order to test the performance of new optimized scheme of mobile IP. The new scheme is simulated using Network Simulator-2 (NS2) version 2.34 [10][11].Network Simulator is a software-based discrete event simulator that runs on Linux based system, and made to support research in networking.

In simulation, FA is put on coordinate of $(600,2)$, and HA on $(1,2)$. The $\mathrm{CN}$ node sends TCP packets stream to $\mathrm{MN}$ and the simulation duration is 250 second. Mobility speed is varied from $10 \mathrm{~m} / \mathrm{s}$, and $20 \mathrm{~m} / \mathrm{s}$. in this simulation 5 nodes was used in network topology, consisting 1 Corresponding Node (CN), 1 Home Agent (HA), 1 Foreign Agent (FA), 1 central router, and 1 Mobile Node (MN). Furthermore, at the time 120 $\mathrm{MN}$ will start move to $\mathrm{FA}$ and leave $\mathrm{HA}$ while $\mathrm{MN}$ still receiving packets, and return back to HA at time 200.

The network model that used in simulation scenario assumed that $\mathrm{CN}$ support mobility functions, and the model consist of two domain and clusters. Moreover, first MN act as node 4 in scenario will receive traffic from $\mathrm{CN}$ which is node 0 in the scenario; when $\mathrm{MN}$ in home network (node 2), it will receive packets according to original MIP operation. If MN moves from it's HA to FA (node 3), then the traffic data flow will take the direct path from $\mathrm{CN}$ to the MN's new location (FA) unlike the original MIP path. This will overcomes the triangle routing problem of the original MIP and enhances the performance by reducing the delay. Figures 5 shows when MN within HA range, and figure 6 shows when $\mathrm{MN}$ within FA range (optimized Mobile IP). 


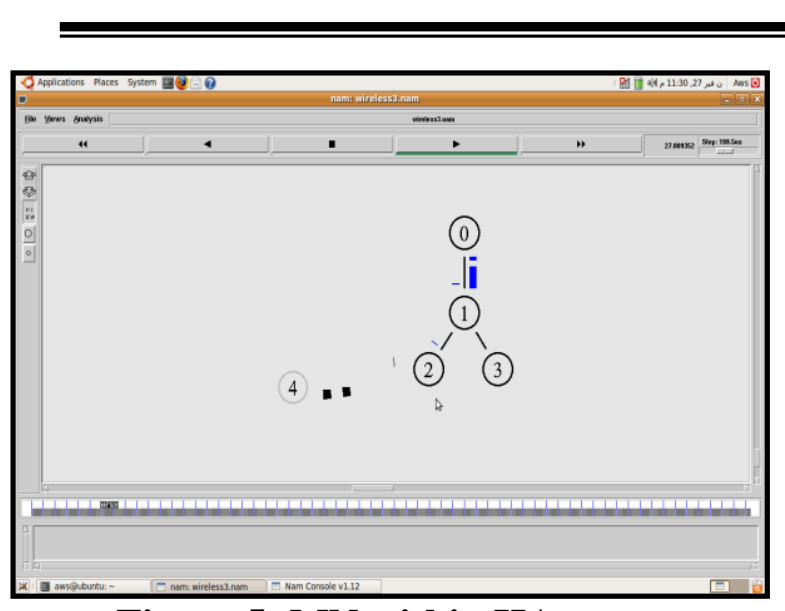

Figure 5: MN within HA range.

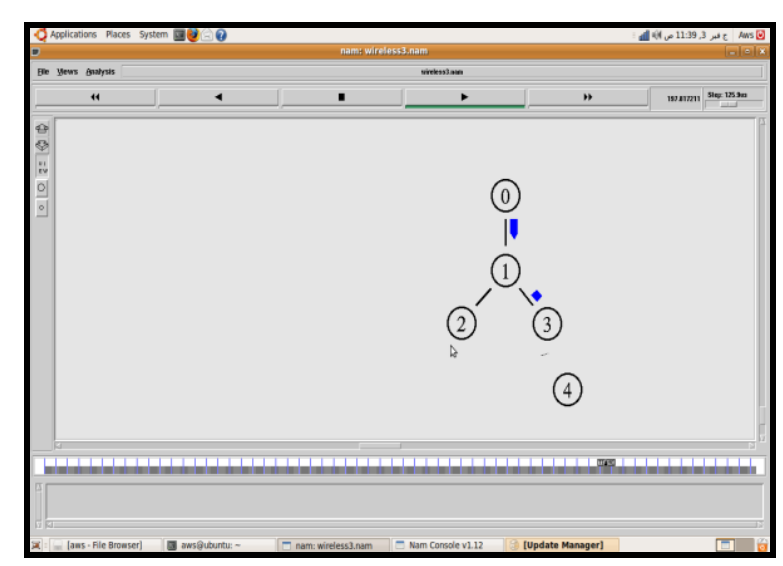

Figure 6: MN within FA ranges (Optimized).

When MN receive packets from its HA while its movement to FA, the packets will pauses in tunneling buffer (TB) until MN reach FA and get new address after that HA will forward the packets to MN. This scheme will reduce packet loss during MN traveling from network to other.

\section{Results and Discussion:}

In order to evaluate the performance of the enhancement technique of Mobile IP and compare it with original Mobile IP and other techniques, the simulation result was used. In this paper two metrics was used to evaluate the performance; Packet Loss and Average End to End Delay.

The packet loss is that packet which sends by $\mathrm{CN}$ to $\mathrm{MN}$ but not reached to its destination and calculate by [12][13].

No. of Packet loss $=$ No. of sending packet - No. of reached packets [14].

Average End to End delay is the time that packet take to travel from its source to its destination, when Average End to End delay is low the performance of the network will be better and calculate by[15].

Avg. end2end delay $=$ Send time - reached time.

The following tables show the results that obtained from simulation on both techniques, original Mobile IP, and optimized Mobile IP. Tables 1 and 2 summarize the results of the simulations and give a better idea about the performance of each scheme in the scenario. It is clear that the proposed scheme performs better in terms of packets loss rate and average end to end delay in scenario.

Table 1: Results of Original Mobile IP

\begin{tabular}{|c|c|c|c|c|}
\hline Time $(\mathrm{Sec})$ & Generated Packets & Received Packed & Loosed Packets & End to End Delay(ms) \\
\hline $1-40$ & 5028 & 2558 & 13 & 8390.14 \\
\hline $40-80$ & 11671 & 5939 & 4 & 13241.6 \\
\hline $80-120$ & 18322 & 9315 & 14 & 18022.6 \\
\hline $120-160$ & 19947 & 10119 & 38 & 19387.6 \\
\hline $160-200$ & 20126 & 13824 & 48 & 24227.9 \\
\hline $200-230$ & 31589 & 16010 & 56 & 31267.6 \\
\hline $230-250$ & 35347 & 18147 & 59 & 33654.8 \\
\hline
\end{tabular}


Propose A New Optimization Technique For Mobile IPv4.

Table 2: Results of Optimized Mobile IP

\begin{tabular}{|c|c|c|c|c|}
\hline Time $(\mathrm{Sec})$ & Generated Packets & Received Packed & Loosed Packets & End to End Delay(ms) \\
\hline $1-40$ & 5028 & 2558 & 13 & 8390.14 \\
\hline $40-80$ & 11671 & 5939 & 4 & 13241.6 \\
\hline $80-120$ & 18322 & 9315 & 14 & 18022.6 \\
\hline $120-160$ & 20932 & 10363 & 26 & 19064.9 \\
\hline $160-200$ & 27043 & 13824 & 21 & 19729.8 \\
\hline $200-230$ & 31060 & 15899 & 28 & 22083.5 \\
\hline $230-250$ & 39944 & 20304 & 33 & 24654.8 \\
\hline
\end{tabular}

Moreover, the packet loss ratio is as shown in figure 7; at the beginning of the simulation is almost has same ratio for both techniques (original and optimized), because $\mathrm{MN}$ receives the sent packets while still it is attached to the Home Agent. When time $=120, \mathrm{MN}$ moving to another subnet then packet loss ratio will increase for original Mobile IP; in case of optimized scheme the packet loss ratio will decrease because it is use tunneling buffer during $\mathrm{MN}$ traveling from its HA to FA. In time $=240$, the packet loss ratio for both techniques will became is almost equal because of MN will travel back to it's HA, and the tunneling buffer will no longer used.

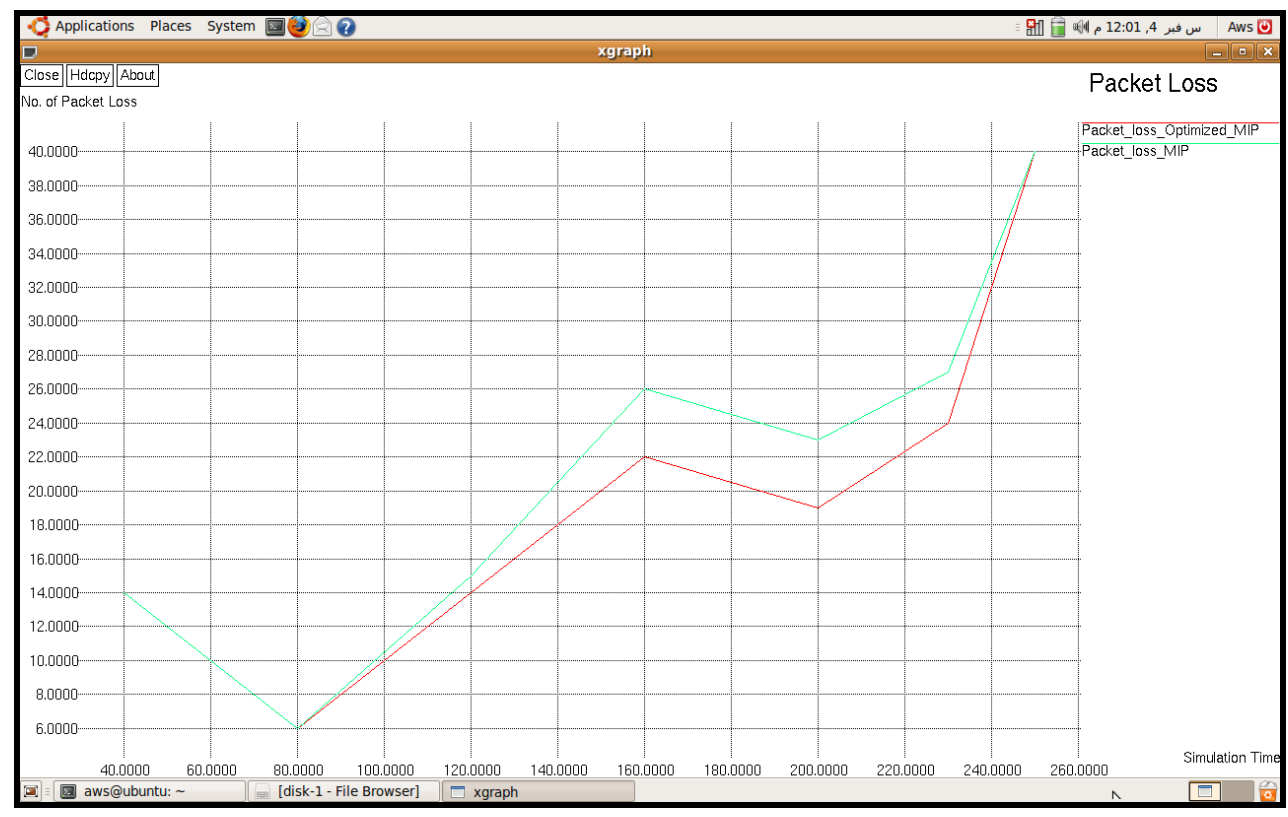

Figure 7: Packet loss ratio.

It can be observe from figure 8 that the average end to end delay for original technique and optimized technique is same; because of $\mathrm{MN}$ is in its HA; till time $120 \mathrm{sec}$ in simulation, on the other hand, when MN start moving and going outside its HA coverage area, the average delay for original technique start increasing. The average delay of the optimized 
schema as same as original schema will increased but with a very low rate compared to the original schema; because the optimized schema will send the packets from $\mathrm{CN}$ to $\mathrm{MN}$ address directly without passing by MN's HA. In original Mobile IP when $\mathrm{MN}$ within FA range, $\mathrm{CN}$ will send the packets stream to MN's HA, and HA will forward the packets to FA then to MN. This will cause delay and packets will be lost. Therefore; the enhanced technique overcome the triangle routing problem in mobile IP.

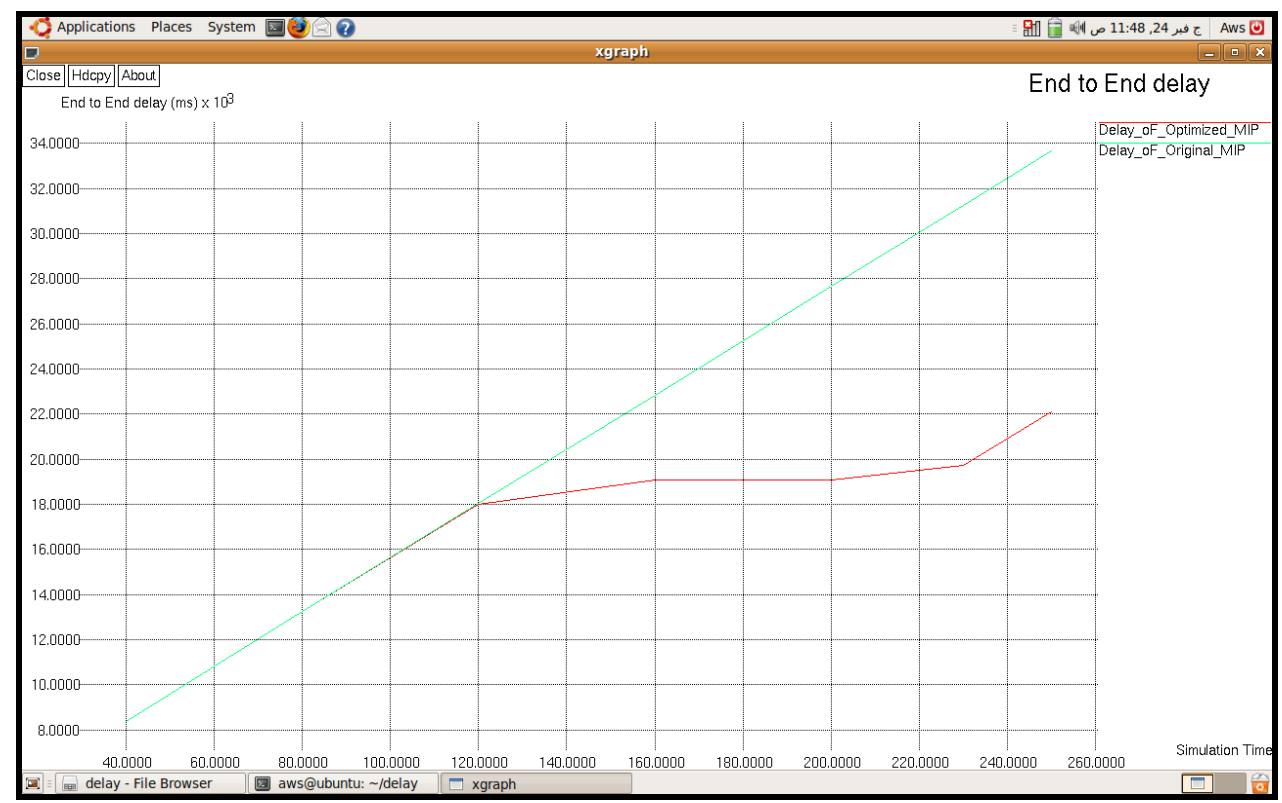

Figure 8: Average end to end delay.

After analyzing the simulation results the proposed scheme shows a very good performance in term of packet loss comparing with ROMIP and Reverse Routing because proposed scheme using tunneling buffer unlike ROMIP and Reverse Routing. On the other hand, the propose technique has similar average end to end delay with ROMIP and Reverse Routing, because all the three techniques will send the packet stream in direct way to FA when MN attached to it and FA forward the packets to MN. On the other words the ROMIP technique has solved the triangle routing problem but in complex way. ROMIP scheme used four kinds of messages and also requires an updates to their binding status. Correspondent nodes $\mathrm{CN}$ have to be Mobility aware to share in Route optimization message exchanges. Thus major hardware and software changes are required in each entity involved [6][11]. On the other hand Reverse Routing is much simpler than the ROMIP one which can be seen from the fact that ROMIP needs four messages whereas Reverse Routing needs only one message. Thus reverse routing have reduced the cost of equipment. However, there is still a problem with reverse routing that Router in the correspondent network $(\mathrm{CN})$ needs to be default router not mobility aware. A simulated comparison between ROMIP and Reverse Routing carried out and Details can be found in [13] [15]. 


\section{Conclusion:}

Mobile IPv4 has some limitations such as triangle routing; many schemes have been proposed to enhance the quality of service in MIPv4 especially during real-time traffic. Each of these schemes has its advantages and disadvantages. This paper proposed a new scheme to reduce real-time traffic packet loss in MIPv4 and average end to end delay. Network simulator ns-2 has been used to simulate the network topology and test the proposed technique. The results of the original mobile IP and proposed scheme have been discussed in detail and a comparison between them was shown as well. Finally, the proposed scheme compare with other schemes (ROMIP and RR).

\section{REFRENCES}

[1] Jamalipour, A., "The Wireless Mobile Internet Archetecture, Protocols and Services", Wiley, (2002).

[2] Perkins, C., "IP Mobility Support for IP v4", RFC 3344, Nokia Research Center, August (2002).

[3] Perkins, C., "Mobile Networking through Mobile IP", Sun Microsystems, February (1998).

[4] Perkins, C. "IP Encapsulation within IP", RFC 2003, May (1996).

[5] T. Janevski, "Traffic Analysis and Design of Wireless IP Networks". Artech House Publishers. Boston, London,(2003).

[6] Perkins, C., "Route Optimization in Mobile IP (ROMIP)", Internet Draft draft-ietf-mobileip-optim-11, August (2002).

[7] Amit Mahajan, Ben Wild, "Route Optimizations In Mobile IP" (2008).

[8] Perkins, C., "Special tunneling", draft-ietf-mobileip-spectun-00, Dec 2000.

[9] Zhou $\mathrm{P}$ et al., "Reverse Routing: An alternative to ROMIP Protocol", University of Ottawa, May 9, (1999).

[10] Greis M., "Tutorial for the UCB/VINT Network Simulator ns", http://www.isi.edu/nsnam/ns/tutorial/index.html.

[11] Mustafa S, Shabbir S, Sardar F, Maqsood A, "Analysis of mobile IP handover latency and packet loss issues using simulation", Comsats institute of information technology, Pakistan, Feb (2011).

[12] Chen H., Trajkovi' L., "Simulation of Route Optimization in Mobile IP", Simon Fraser University, Burnaby, BC, Canada, (2010).

[13] Pang et al., "Comparison of Route Optimization and Reverse Routing", Final Report, Fall (2003).

[14] R. Timothy, Stuedi P, "Advance computer network wireless/ Mobility", System ETH, (25 march 2012).

[15] Brian E. Carpenter, Moore K, Fink B, "Routing IPv6 over IPv4", The Internet Protocol Journal - Volume 3, No. 1 (2011). 